Res., Soc. Dev. 2019; 8(2):e1282585

ISSN 2525-3409 | DOI: http://dx.doi.org/10.33448/rsd-v8i2.585

\title{
Uma revisão bibliométrica sobre a co-pirólise de biomassa e resíduo plástico
}

A bibliometric review on a co-pyrolysis of biomass and plastic waste

Una revisión bibliométrica sobre la co-pirólisis de biomasa y residuo plástico

Felipe Santos Pimentel

ORCID: https://orcid.org/0000-0002-4815-9649

Universidade Federal do Espírito Santo, Brasil

E-mail: felipesantos.pimentel@hotmail.com

Thiago Padovani Xavier

ORCID: https://orcid.org/0000-0001-7148-9921

Universidade Federal do Espírito Santo, Brasil

E-mail: thiago.p.xavier@ufes.br

Taisa Shimosakai de Lira

ORCID: https://orcid.org/0000-0002-2690-242X

Universidade Federal do Espírito Santo, Brasil

E-mail: taisa.lira@ufes.br

Recebido: 14/08/2018 - Aceito: 30/08/2018

\section{Resumo}

O crescimento econômico desafia a seguridade da matriz energética em suprir a demanda por energia da sociedade. Além dos riscos ambientais, os combustíveis fósseis estão cada vez menos capazes de suprir esta demanda, fazendo com que pesquisas por fontes renováveis sejam alavancadas. Dentro deste contexto, os recentes estudos sobre a co-pirólise, técnica que promove a diminuição do volume de resíduos e geração de compostos de alto valor agregado, estão ganhando notoriedade. Tendo como objetivo a avaliação da maturidade destes estudos e a formação de um banco de dados específico sobre o tema, utilizou-se como ferramenta a revisão bibliométrica, esta que permite a seleção de artigos dentro de um banco de dados a partir de parâmetros estatísticos, como número de citações e fator de impacto. Portanto, podese concluir que apesar de recente, a co-pirólise se apresenta como uma técnica promissora, simples e de baixo custo, para o melhoramento do rendimento e composição estrutural dos produtos de pirólise.

Palavras-chave: Energia; Fontes renováveis; Matriz Energética. 


\section{Abstract}

Economic growth challenges the security of the energy matrix in meeting the energy demand of society. In addition to environmental risks, fossil fuels are increasingly unable to meet this demand, and research by renewable sources is leveraged. Within this context, recent studies on co-pyrolysis, a technique that promotes the reduction of waste volume and the generation of compounds with high value, are gaining notoriety With the objective of evaluating the maturity of these studies and the formation of a specific database on the subject, a bibliometric revision was used as a tool, which allows the selection of articles within a database based on statistical parameters, such as number of citations and impact fator. Therefore, it can be concluded that, although recent, co-pyrolysis presents as a promising technique, simple and low cost, for the improvement of the yield and structural composition of pyrolysis products.

Keywords: Energy; Renewable source; Energetic matrix.

\section{Resumen}

El crecimiento económico desafía la seguridad de la matriz energética en suplir la demanda de energía de la sociedad. Además de los riesgos ambientales, los combustibles fósiles están cada vez menos capaces de suplir esta demanda, haciendo que las investigaciones por fuentes renovables sean apalancadas. En este contexto, los recientes estudios sobre la co-pirólisis, técnica que promueve la disminución del volumen de residuos y la generación de compuestos de alto valor agregado, están ganando notoriedad. Con el objetivo de evaluar la madurez de estos estudios y la formación de un banco de datos específico sobre el tema, se utilizó como herramienta la revisión bibliométrica, esta que permite la selección de artículos dentro de una base de datos a partir de parámetros estadísticos, como número de citas y factor de impacto. Por lo tanto, se puede concluir que a pesar de reciente, la co-pirólisis se presenta como una técnica prometedora, simple y de bajo costo, para el mejoramiento del rendimiento y composición estructural de los productos de pirólisis.

Palabras clave: Energía; Fuentes renovables; Matriz energética.

\section{Introdução}

Frente ao crescimento econômico, a demanda energética se torna insustentável quando suprida apenas por fontes fósseis (Wong et al., 2015). Esta deficiência energética em conjunto a problemas ambientais, causados pelo uso intenso de combustíveis fósseis, impulsionam a 
busca por fontes energéticas renováveis.

Tratamentos térmicos para geração de energia estão sendo amplamente estudados. Neste contexto, a co-pirólise surge como alternativa na produção compostos de maior valor agregado, como combustíveis e compostos de síntese, e melhoramento destes produtos. Entre as vantagens da utilização da co-pirólise estão a redução no volume de resíduos, geração de compostos de síntese para indústria química e geração de energia (Marin et al., 2002).

Segundo o IPEA (2012) a composição gravimétrica média dos resíduos sólidos urbanos no Brasil possui 13,5\% de plásticos e 51,4\% de matéria orgânica e de acordo com a ABRELPE (2015) foram gerados 79,9 milhões de toneladas de resíduos sólidos urbanos no ano de 2015.

A Biomassa se destaca como uma forte alternativa para geração de energia devido a sua abundância, seu baixo custo e por ser uma fonte renovável (Çepelioğullar e Pütün, 2013). Porém o líquido (bio-óleo) formado a partir da pirólise de biomassa possui baixo poder calorífico, devido ao alto teor de compostos oxigenados em sua estrutura (Wong et al., 2015).

Em contrapartida, os plásticos possuem baixo teor e oxigênio em sua composição estrutural. Com produção aproximada de 5,8 milhões de toneladas no ano de 2016 (ABIPLAST, 2016), e não efetividade de tratamento usando os métodos tradicionais, como aterramento e incineração, uma alternativa pouco viável devido à falta de espaço, aumento de custos e a geração de gases tóxicos e de efeito estufa (Çepelioğullar e Pütün, 2013, Paradela et al., 2008), a pirólise apresenta, neste contexto, uma alternativa viável para gerenciamento de resíduos através de menores realocações de esforços como demanda por espaço ou rearranjo da logística espacial.

Os benefícios da alimentação em conjunto de biomassa e resíduos plásticos são expressivos, como em Önal et al. (2012) onde a copirólise de casca de batata e polietileno de alta densidade (PEAD) promoveu maior produção de bio-óleo, com o aumento da porcentagem PEAD na alimentação, e melhoria na composição estrutural do produto, maiores teores de hidrogênio e carbono e menor teor de oxigênio. Em Çepelioullar e Pütun (2014), a pirólise lenta da mistura de biomassa e plásticos, promoveram melhorias estruturais nos produtos líquido e gasoso. Sajdak e Muzyka (2014), observaram que a adição de polipropileno reduz a energia necessária para a conversão térmica da biomassa.

Durante o coprocessamento, os plásticos servem como doadores de hidrogênio para a biomassa, elevando a razão hidrogênio/carbono e consequentemente elevando o poder calorífico do produto final (Brebu et al., 2010). O parâmetro de maior significância na operação é a razão de mistura das matérias primas, durante a alimentação (Cornelissen et al., 
2008).

Como forma de avaliar a maturidade em que se encontram as pesquisas sobre o tema co-pirólise de biomassa e plásticos, pode-se utilizar uma ferramenta estatística como a revisão bibliométrica. Esta que permite a imersão sobre as pesquisas de maior impacto intelectual no campo desejado a partir de dados estatísticos. Caracterizada como um método que possibilita a coleta e avaliação crítica de dados (Botelho et al., 2011). Utilizada para investigação de lacunas e direcionamento de pesquisas com maior precisão, consequentemente, minimizando a probabilidade de erros nas decisões para resolução de problemas (Macedo et al., 2010).

Este trabalho tem como objetivo a avaliação da maturidade das pesquisas sobre o tema co-pirólise e a formação de um banco de dados específico.

\section{Metodologia}

A pesquisa foi realizada na plataforma Scopus, que possui em sua biblioteca somente artigos que estão em sua versão final de publicação. A formação do banco de dados foi conduzida em 3 passos, como ilustra o fluxograma da Figura 1. Os dois primeiros passos foram exploratórios, onde foi possível analisar os principais ramos da pesquisa em função de aplicação e estado da arte, gerando um banco de dados extenso. E, posteriormente, no terceiro passo, foi feita a especificação do banco de dados em função do trabalho a ser desenvolvido.

Figura 1 - Fluxograma da formação do banco de dados de artigos específicos.

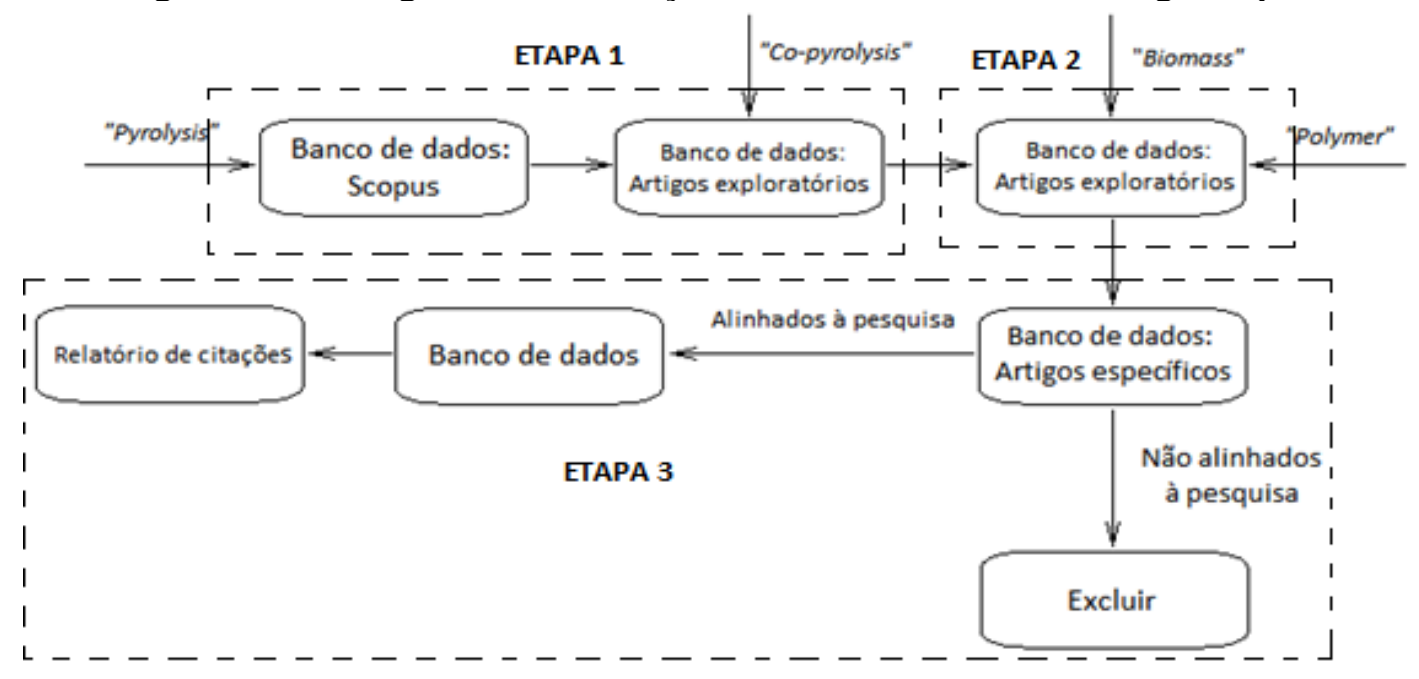

Fonte: Elaborado pelo autor.

A chave de pesquisa foi inicialmente "Pyrolysis", onde abordou-se todo o seu histórico e aplicações. Em seguida foi acrescentado a chave "Co-pyrolysis", em que mesmo 
em caráter exploratório, de forma a averiguar suas principais aplicações e segmentos utilizados, foi possível relacionar ao tema principal deste trabalho. E, por fim, o direcionamento da pesquisa foi dado com a implementação de duas novas chaves, "Biomass" e "Polymer", onde reduziu-se o número de artigos de forma a enquadrar somente aqueles que possuíssem uma estreita relação com o tema do trabalho a ser realizado.

A partir dos critérios de busca mencionados, gerou-se o banco de dados específico à pesquisa, onde foram analisados o número de publicações por ano, o número de publicações por países, o índice-h e o número de citações por ano.

Os artigos foram listados de forma decrescente em função do número de citações, de modo que, ao final, se dispusessem os artigos mais relevantes à pesquisa da co-pirólise de biomassa e resíduo plástico.

\section{Resultados e discussão}

Partindo do banco de dados da plataforma Scopus, a utilização da chave de pesquisa "Pyrolysis" gerou um total 108.455 artigos (Figura 2), em que o primeiro artigo publicado foi no ano de 1923, no periódico Industrial and Engineering Chemistry, por McKee e Goodwin.

Figura 2 - Número de publicações com a chave "pyrolysis” em função do ano.

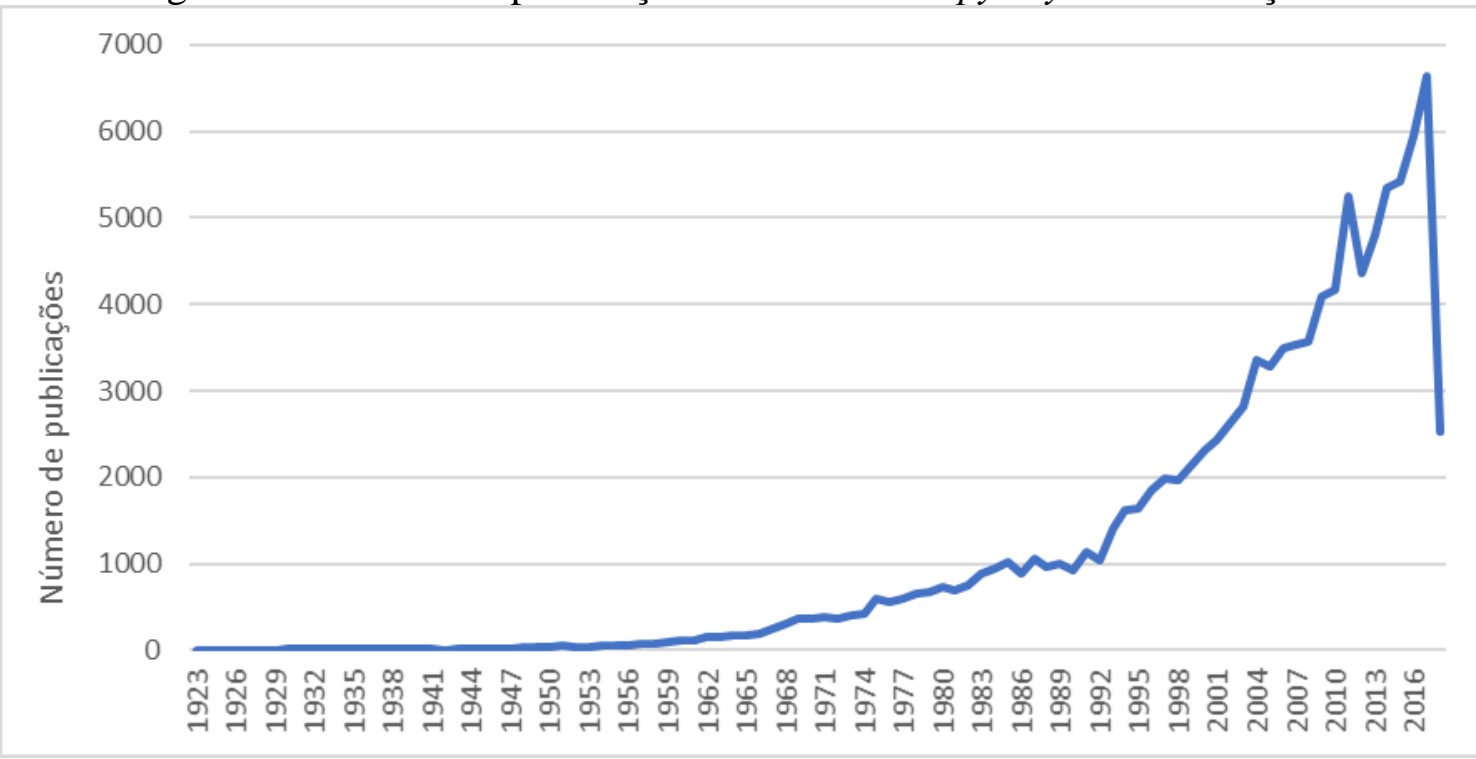

Fonte: Elaborado pelo autor.

Apesar das publicações sobre pirólise se iniciarem em 1923, a quantidade de estudos sobre esta temática tornou-se significativa a partir de 1949, onde o número de publicações foi 
ISSN 2525-3409 | DOI: http://dx.doi.org/10.33448/rsd-v8i2.585

de 38, enquanto nos anos anteriores alcançavam no máximo 28. Em 2017, houve a maior número de publicações, 6.650. Os 10 países com maior destaque em relação ao número de publicações são mostrados na Figura 3, tendo a China e Estados Unidos na liderança. O Brasil está na $14^{\mathrm{a}}$ colocação.

Figura 3 - Número de publicações por país, com a chave "pyrolysis".

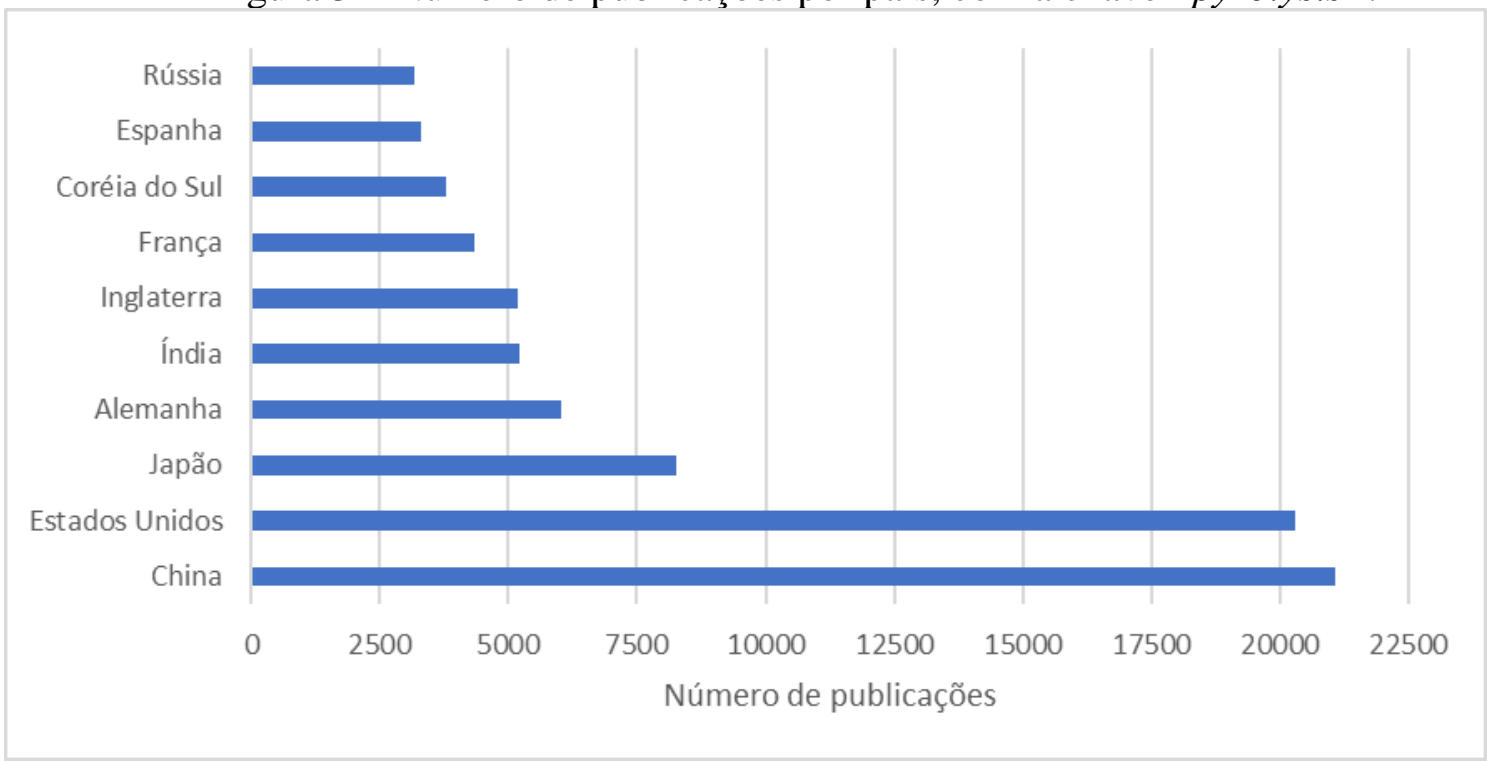

Fonte: Elaborado pelo autor.

As duas maiores afiliações relacionadas ao tema pirólise são Chinesas, Academia Chinesa de Ciências e Ministério de Educação da China, o que demonstra o grande interesse do país sobre o tema e sua importância. O autor com maior número de publicações é Yun Chan Kang e a fonte com maior número de publicações é a Journal Of Analitycal And Applied Pyrolysis.

Foi realizada a leitura dos 10 artigos mais citados (Tabela 1). Em sua maioria são reviews sobre as possíveis aplicações da pirólise.

Tabela 1 - Artigos mais citados com a chave "pyrolysis".

\begin{tabular}{cc}
\hline Título & Autores \\
\hline Biodiesel production: a review & Ma e Hanna (1999) \\
\hline Pyrolysis of wood/biomass for bio-oil: a critical review & Mohan et al. (2006) \\
\hline $\begin{array}{c}\text { Characteristics of hemicellulose, cellulose and lignin } \\
\text { pyrolysis }\end{array}$ & Yang et al. (2007) \\
\hline
\end{tabular}

Iron-based catalyst with improved oxygen reduction activity in polymer electrolyte fuel cells

Lefèvre et al. (2009)

Overview of applications of biomass fast pyrolysis oil Czernik e Bridgwater (2004) 
A new analysis of thermogravimetric traces

Chemical kinetic data base for combustion chemistry. Part I. Methane and related compounds
Horowitz e Metzger (1963)

Tsang e Hampson (1986)

Review of fast pyrolysis of biomass and product upgrading

Bridgwater (2012)

Comparison of the Mechanism of Toxicity of Zinc Oxide and Cerium Oxide Nanoparticles Based on Dissolution and Oxidative Stress Properties

Xia et al. (2008)

An overview of hydrogen production Technologies

Holladay et al. (2009)

Fonte: Elaborado pelo autor.

Ma e Hanna (1999) realizaram um review sobre a produção de biodiesel sob diversos métodos, dentre eles, a pirólise a qual indicam a produção de compostos similares à gasolina e diesel.

Mohan et al. (2006) fizeram uma análise sobre a pirólise rápida de biomassa vegetal para produção de bio-óleo, que possuem fins de fonte energética e matéria-prima para indústria química, dissertando sobre os efeitos da composição e estrutura do composto, taxa de aquecimento e tempo de residência.

Yang et al. (2007) investigaram as características da pirólise dos três principais componentes de biomassas: hemicelulose, celulose e lignina. Revelando as faixas de temperatura em que os materiais sofrem decomposição, sendo 493,15 - 588,15 K a hemicelulose e 588,15 - 673,15 K a celulose, já a lignina possui uma ampla faixa térmica para sua decomposição, 433,15 - 1173,15 K. Foi encontrado que para hemicelulose, o maior rendimento de produto gasoso foi o $\mathrm{CO} 2$, para a celulose, o $\mathrm{CO}$, e para lignina, $\mathrm{H}_{2}$ e $\mathrm{CH}_{4}$.

Lefèvre et al. (2009) aplicaram a pirólise para perda de massa em temperaturas acima de $1073 \mathrm{~K}$ na produção de catalisadores a base de ferro, com maiores números de sítios ativos por unidade de volume. A perda de massa em que se obteve a maior atividade foi cerca de 30 $-50 \%$, em peso.

Czernik e Bridgwater (2004) discorrem sobre uma visão geral da produção de bio-óleo a partir da pirólise rápida, salientando suas vantagens de estocagem e transporte. Indicando que já foram realizados testes de suas aplicações em engrenagens, turbinas e caldeiras, embora seus custos energéticos e financeiros ainda considerados inaceitáveis.

Horowitz e Metzger (1963) introduziram uma nova interpretação matemática dos dados termogravimétricos, permitindo a determinação de parâmetros cinéticos das reações de pirólise. Validando o método através da comparação com os dados fornecidos na literatura. 
ISSN 2525-3409 | DOI: http://dx.doi.org/10.33448/rsd-v8i2.585

Tsang e Hampson (1986) avaliaram dados das propriedades cinéticas e termodinâmicas de compostos que são importantes na pirólise e combustão do metano, onde todas as possíveis reações foram consideradas, formando um banco de dados. Em que foram consideradas faixas de temperatura e densidade de $300-2500 \mathrm{~K}$ e $1016-1021$ moléculas $/ \mathrm{cm}^{3}$, respectivamente.

Bridgwater (2012) faz um review atualizado sobre a pirólise rápida e melhoramento do bio-óleo, descrevendo os tipos de reatores, influência da taxa de aquecimento, remoção do carvão e coleta de líquido, características do bio-óleo, melhoramento do bio-óleo por métodos físicos e químicos, aplicações do bio-óleo e fatores econômicos, como custo total para implementação de um sistema de pirólise rápida.

Xia et al. (2008) utilizaram a pirólise para produção de nanopartículas de óxidos metálicos.

Holladay et al. (2009) realizam um review sobre as tecnologias de produção de hidrogênio, incluindo pirólise, através de fontes fósseis ou renováveis.

Ao refinar a pesquisa com a introdução de uma nova chave: "Co-pyrolysis", foram encontrados 984 arquivos, datados entre 1961 a 20 de abril de 2018, com primeira publicação de 1961 no periódico Journal of the Chemical Society, por Birchall, Haszeldine e Parkinson. Como pode ser visto na Figura 4, o ano de maior destaque em foi o de 2017 com 147 publicações.

Figura 4 - Número de publicações por ano, com adição da chave "co-pyrolysis".

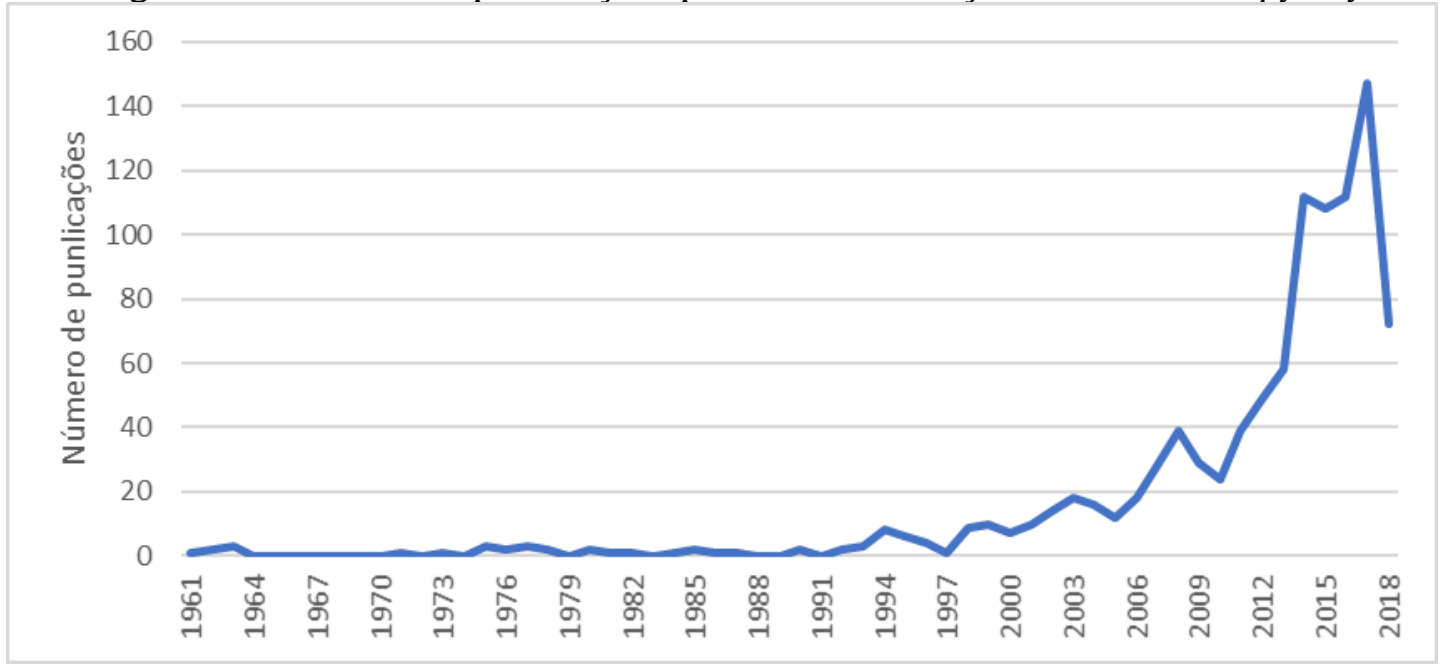

Fonte: Elaborado pelo autor.

$\mathrm{Na}$ figura 5 são apresentados os 10 países com maiores volumes de publicações relacionados a figura 4. Novamente, a China possui liderança absoluta no número de 
publicações, sendo que o Brasil aparece na $22^{\mathrm{a}}$ colocação.

Figura 5 - Número de publicações por país, com adição da chave "co-pyrolysis".

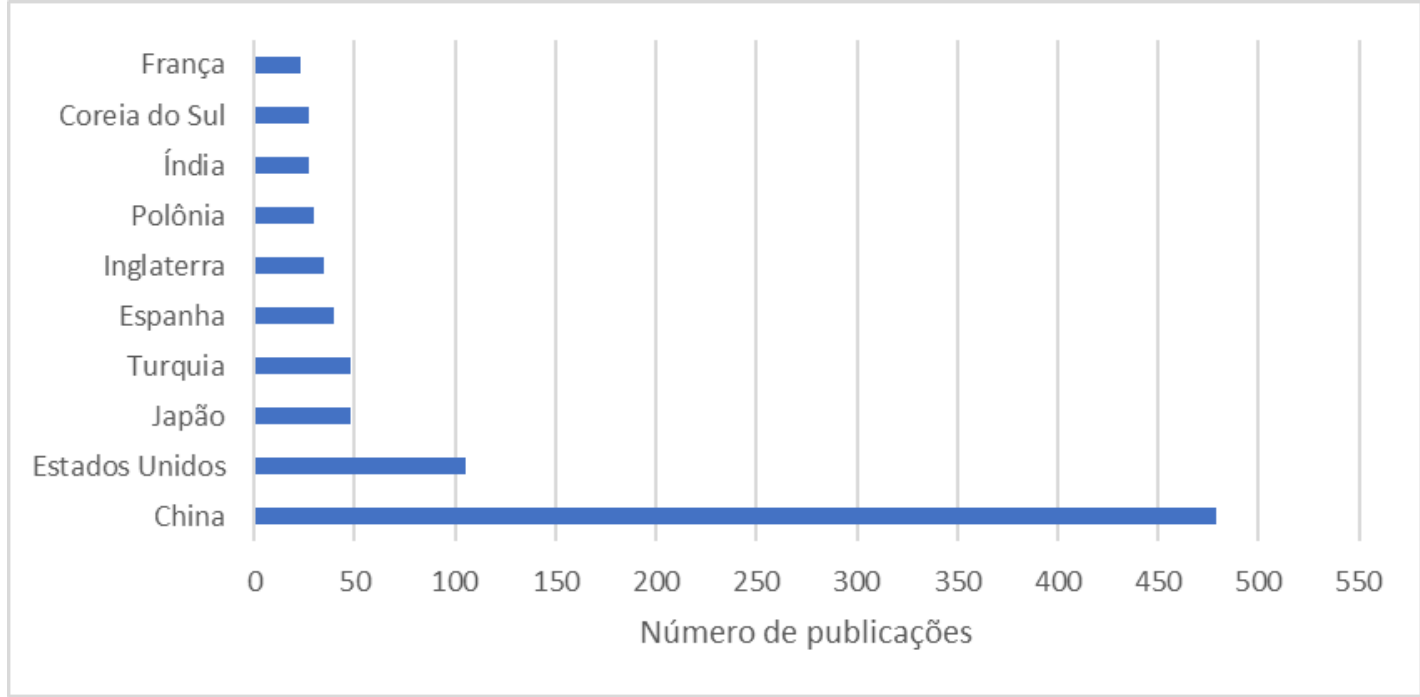

Fonte: Elaborado pelo autor.

As afiliações de maior apoio são novamente o Ministério de Educação da China e a Academia Chinesa de Ciências, reforçando a importância da produção de energia renovável para o país. O autor de maior publicação foi Ma Xiaoqian e o periódico em que houve maior número de publicações foi novamente o Journal Of Analytical And Applied Pyrolysis. E, por fim, os 10 artigos mais citados após a adição da chave "Co-pyrolysis" está na Tabela 2.

Tabela 2 - Artigos mais citados, com adição da chave "co-pyrolysis".

\section{Título}

Co-pyrolysis and co-gasification of coal and biomass in bech-scale fixed-bed and fluidised bed reactors
Autores

Collot et al. (1999)

Thermogravimetric characteristics and kinetic of plastic and biomass blends co-pyrolysis

Zhou et al. (2006)

Co-pyrolysis of wood biomass and synthetic polymer mixtures. Part I: influence of experimental conditions on the Sharypov et al. (2002) evolution of solids, liquids and gases

Co-pyrolysis of sugarcane bagasse with petroleum residue.

Part I: thermogravimetrics analysis

Garcìa-Pèrez et al. (2001) 
Co-pyrolysis characteristics of sawdust and coal blend in TGA and fixed bed reactor

Park et al. (2010)

Co-pyrolysis of biomass and coal in a free fall reactor

Zhang et al. (2007)

Catalytic gasification of char from co-pyrolysis of coal and biomass

Zhu et al. (2008)

Synergetic effect of during co-pyrolysis/gasification of biomass and sub-bituminous coal

Krerkkaiwan et al. (2013)

Co-pyrolysis of pine cone with sinthetic polymers

Brebu et al. (2010)

Thermogravimetric analysis and kinetics of co-pyrolysis of raw/torrefied wood and coal blends

Lu et al. (2013)

Fonte: Elaborado pelo autor.

Collot et al. (1998) realizaram a co-pirólise e co-gaseificação de carvão e biomassa em reatores, leito fixo e fluidizado, não encontrando efeito sinérgico acentuado.

Zhou et al. (2006) investigaram o comportamento da co-pirólise de serragem da madeira de pinho chinês com polietileno de alta e baixa densidade e polipropileno, evidenciando o efeito sinérgico através da avaliação da perda de massa da mistura, cerca de 6 $-12 \%$ a $803,15-923,15 \mathrm{~K}$.

Sharypov et al. (2002) avaliaram a co-pirólise de diversos tipos de biomassa e polímeros plásticos, encontrando a temperatura para produção de líquidos leves à $673,15 \mathrm{~K}$ e que as origens de ambas matérias-primas possuem forte influência na co-pirólise.

Garcìa-Pèrez et al. (2001) estudaram a co-pirólise de bagaço de cana e resíduo de petróleo através da termogravimetria, não encontrando interação significante na fase sólida da mistura.

Park et al. (2010) analisaram as características da co-pirólise da mistura de serragem e carvão, encontrando que a máxima sinergia foi na razão de 0,6 de serragem à 873,15 K. Esta sinergia promoveu a formação de maior quantidade de voláteis que se comparado a soma do rendimento da pirólise de cada material separadamente.

Zhang et al. (2007) realizaram a co-pirólise entre biomassa e carvão, onde foi verificado a existência de efeito sinérgico a partir do aumento do rendimento de líquido e consequentemente houve diminuição de carvão.

Zhu et al. (2008) verificaram que o carvão produzido a partir da co-pirólise possuía maior reatividade de gaseificação que o carvão produzido a partir da pirólise pura. 
ISSN 2525-3409 | DOI: http://dx.doi.org/10.33448/rsd-v8i2.585

Krerkkaiwan et al. (2013) avaliaram o efeito sinérgico entre biomassa e carvão, resultando um aumento no rendimento de produto gasoso e diminuições dos rendimentos do carvão e alcatrão, manifestado principalmente na razão de 1:1.

Brebu et al. (2010) aplicaram a co-pirólise de pinho com três diferentes tipos de polímeros plásticos, polietileno, polipropileno e poliestireno, para investigar o efeito da natureza da biomassa e plástico no rendimento e qualidade dos produtos, carvão e bio-óleo. Foi encontrado que a composição do bio-óleo depende do tipo de polioleofina utilizada. $\mathrm{O}$ poder calorífico do carvão gerado a partir da co-pirólise é maior que o gerado a partir da pirólise separada da biomassa.

Lu et al. (2013) avaliaram o comportamento térmico e a cinética de co-pirólise entre madeira crua/queimada com carvão, utilizando 5 diferentes composições da mistura. Os resultados das análises sugerem que as características da co-pirólise são muito próximas das características da combinação dos materiais individuais. Portanto, o efeito sinérgico entre a biomassa e o carvão são pouco significativos.

Após duas pesquisas exploratórias e consequente formulação de um banco de dados extenso, na terceira etapa foi realizada a inserção de chaves específicas de procura, buscando refinar os artigos para criação de um banco de dados específico. Adicionando "Biomass" e "Polymer”, o número de artigos foi reduzido a 51 artigos no período de 2002 a 2018 (Figura $6)$.

Figura 6 - Número de publicações por ano, com adição das chaves "biomass" e "polymer".

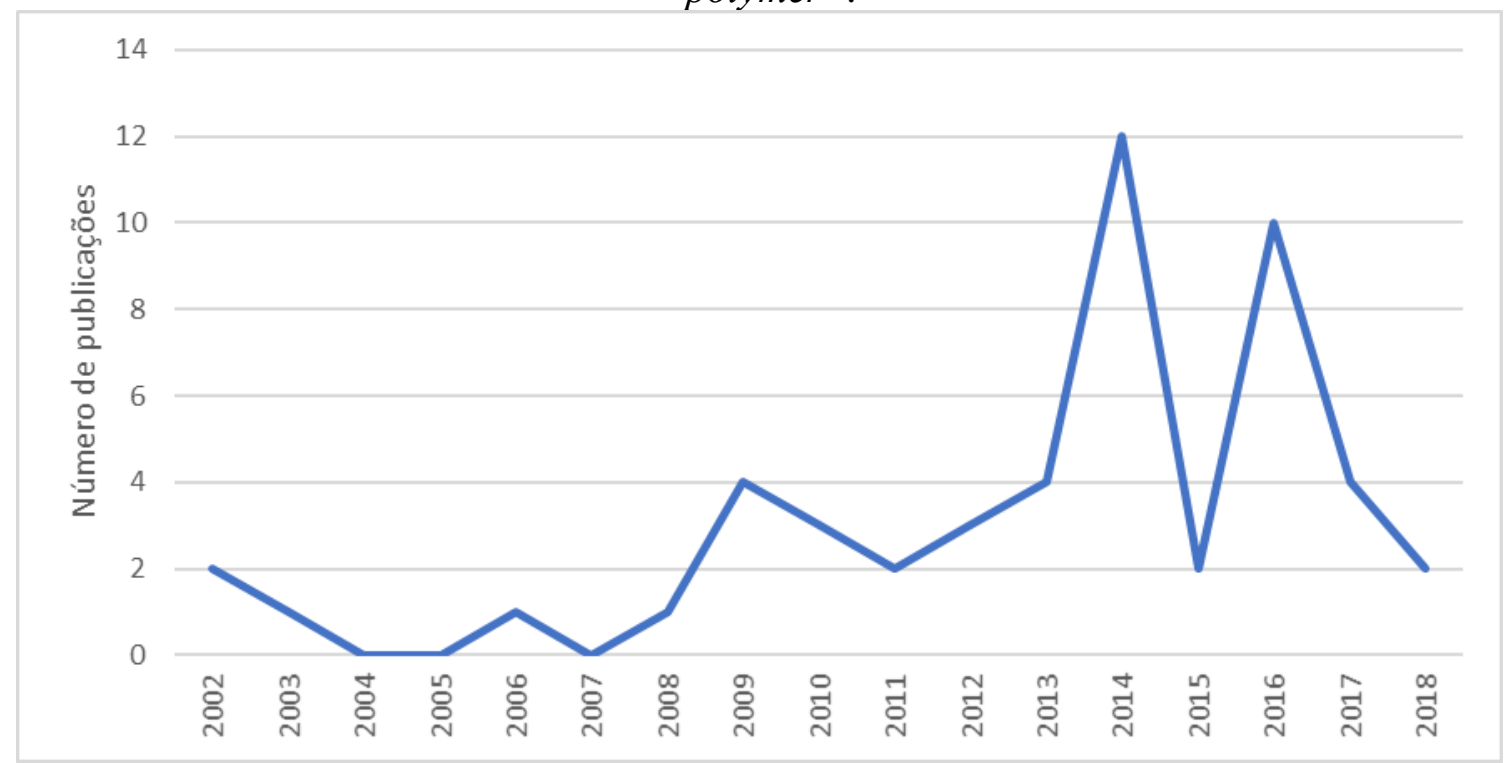

Fonte: Elaborado pelo autor. 
Res., Soc. Dev. 2019; 8(2):e1282585

ISSN 2525-3409 | DOI: http://dx.doi.org/10.33448/rsd-v8i2.585

O ano de 2014 houve o maior pico de publicações (12) e dentre os 10 países com maior número de publicações, novamente, destaca-se a China (Figura 7). Destaca-se também o fato de que o Brasil não possui nenhuma publicação dentro do tema abordado.

Figura 7 - Número de publicações por país, com adição das chaves "biomass" e "polymer".

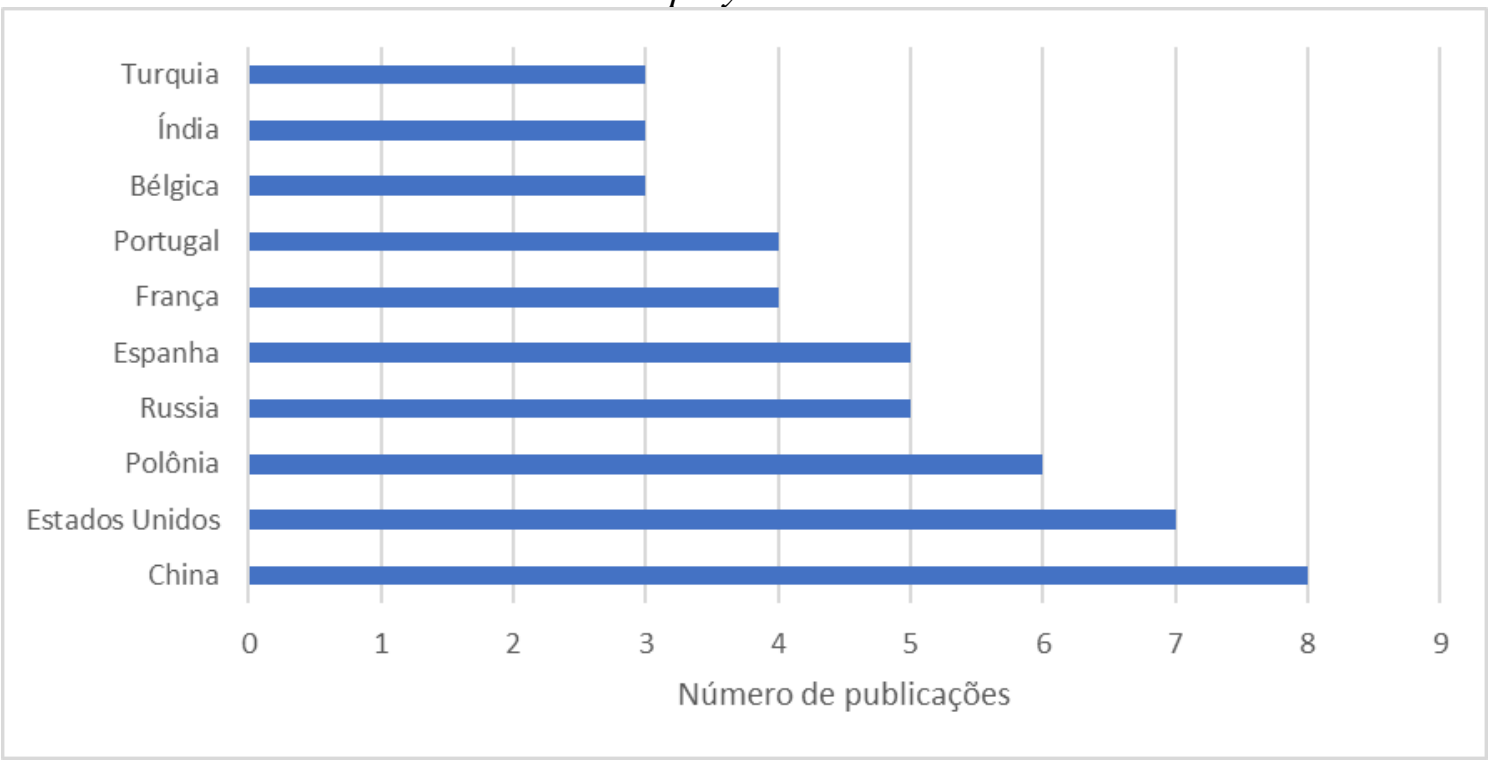

Fonte: Elaborado pelo autor.

O autor com maior número de publicações foi Natalia G. Beregovtsova, o periódico em destaque foi novamente o Journal Of Analytical And Applied Pyrolysis e a afiliação de maior investimento foi o Consejo Superior de Investigaciones Científicas, uma agência do estado espanhol.

Após o refinamento da pesquisa, o índice-h do banco de dados foi de 21, o número de citações por ano é mostrado na Figura 8 e os 10 artigos mais citados (que possuem maior relevância para este trabalho) estão na Tabela 3.

Figura 8 - Número de citações por ano.

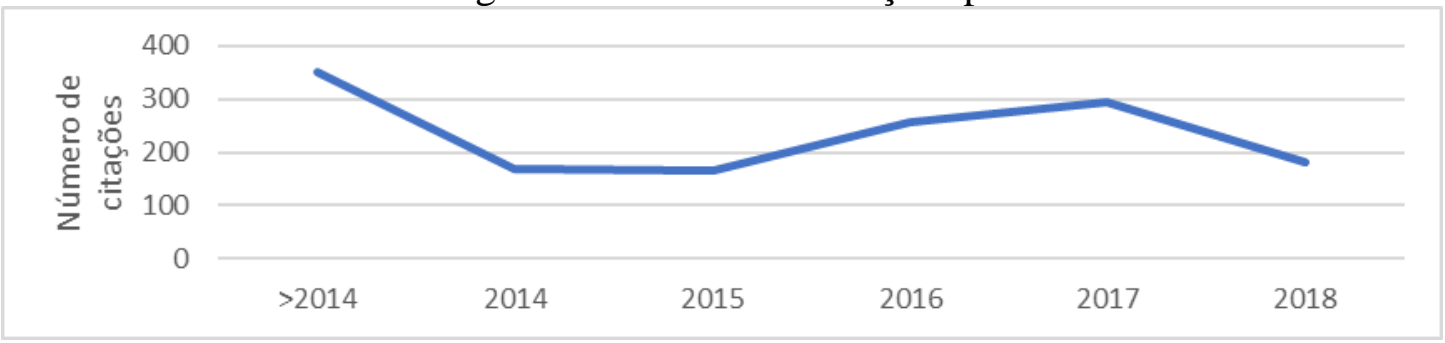

Fonte: Elaborado pelo autor. 
Tabela 3 - Artigos mais citados, com adição das chaves "biomass" e "polymer".

\section{Título}

Co-pyrolysis of wood biomass and synthetic polymer

mixtures. Part I: influence of experimental conditions on evolution of solids, liquids and gases
Autores

Co-pyrolysis of pine cone with synthetic polymers

Brebu et al. (2010)

Co-pyrolis of wood biomass and synthetic polymer

mixtures. Part II: characterisation of the liquid phases

Marin et al. (2002)

Co-pyrolysis of wood biomass and synthetic polymer

mixtures. Part III: characterisation of heavy products

Sharypov et al. (2003)

Thermal and kinetic behaviors of biomass and plastic wastes in co-pyrolysis

Çepelioğullar e Pütün (2013)

Dorado et al. (2014)

H-ZSM5 catalyzed co-pyrolysis of biomass and plastics

Önal et al. (2014)

Bio-oil production via co-pyrolysis of almond shell as biomass and high density polyethylene

Cornelissen et al. (2008)

Flash co-pyrolysis of biomass with polylactic acid. Part I: influence of bio-oil yield and heating value

Wong et al. (2015) source of fuel: A review

Paradela et al. (2009)

Study of co-pyrolysis of biomass and plastic wastes

Fonte: Elaborado pelo autor.

Sharypov et al. (2002) e Brebu et al. (2010) já citados anteriormente demonstram um grande impacto sobre o assunto mesmo em pesquisas exploratórias e de caráter mais abrangente;

Marin et al. (2002) realizam a segunda parte da discussão abordada por Sharypov et al. (2002). Fizeram a caracterização das fases líquidas, onde se obteve 50\%, em massa, de líquido, concluindo que a biomassa, independente da origem, guia a produção de sólido, água e gás, enquanto o polímero guia a produção de líquido, olefinas gasosas e parafinas.

Sharypov et al. (2003) complementaram com terceira parte da discussão em que o tema abordado é a caracterização dos produtos pesados em técnicas avançadas, tais como FTIR e GC-MS, discutindo também algumas perspectivas da conversão térmica do polímero e biomassa. 
Çepelioğular e Pütün (2013) investigaram as características da co-pirólise, de diversos tipos de biomassa e polímeros plásticos, e sua cinética, indicando que as diferenças estruturais afetam diretamente seu comportamento na degradação térmica. Concluíram que a adição de materiais plásticos à pirólise de biomassa modifica não somente seu comportamento térmico, mas seu comportamento cinético.

Dorado et al. (2014) realizaram a co-pirólise de biomassa e plásticos utilizando catalizador H-ZSM5, utilizando como biomassa, resíduos agrícolas. Os resultados indicam aumentos no total de compostos aromáticos quando as misturas de biomassa e plásticos são submetidas a co-pirólise rápida na presença de catalisador H-ZSM5.

Önal et al. (2014) observaram um aumento de $23 \%$ do rendimento de líquido quando a massa de polietileno de alta densidade dobrou, em sua mistura com casca de amêndoa.

Cornelissen et al. (2008) utilizaram a co-pirólise de salgueiro e ácido polilático para redução do teor de água no bio-óleo produzido, em que a proporção 1:2 de salgueiro/Ácido obteve a sinergia mais expressiva, com redução de teor de água em 37\% no bio-óleo.

Wong et al. (2015) descrevem sobre o estado atual e as perspectivas de resíduos plásticos como fontes de energias, destacando a co-pirólise de biomassa e plásticos.

Paradela et al. (2009) estudaram os efeitos das condições experimentais no rendimento e composição dos produtos, na co-pirólise de pinho e resíduos plásticos. Observando que o aumento do tempo de reação no produto gasoso aumentou a quantidade de alcanos e no produto líquido aumentou a quantidade de aromáticos. $\mathrm{O}$ aumento na temperatura de reação diminuiu o rendimento de produtos líquidos com consequente aumento dos produtos gasosos e sólidos. Por fim, aumento de pressão inicial nos líquidos aumentou os compostos aromáticos e no gasoso favoreceu a formação de monóxido e dióxido de carbono.

\section{Considerações finais}

Por meio desta revisão bibliométrica, foi possível aprofundar o conhecimento sobre a co-pirólise, avaliando o crescimento do número de publicações, os países de maior enfoque e os órgãos afiliados. Disponibilizando um acervo textual de significante impacto literário.

Concluiu-se que pode haver efeito sinérgico ou não, e com maior ou menor grau de interação entre as matérias-primas, a depender do tipo de biomassa e plástico utilizados. Foi verificado que o produto final depende de uma série de parâmetros operacionais, bem como a origem e estrutura que compõe a biomassa e resíduo plástico. $\mathrm{O}$ foco dos trabalhos, em geral, realizados até então se dá no melhoramento do produto final, seja ele líquido, sólido ou 
gasoso, mas principalmente o bio-óleo.

Portanto, apesar de recente, a técnica de co-pirólise apresenta um grau elevado de maturidade de pesquisa, onde abrangem de forma vasta os parâmetros operacionais aplicados, suas interferências e suas implicações sobre os resultados. Deixando em aberto o estudo com aplicação de novas matérias-primas, correlações entre os parâmetros operacionais, aplicação de diferentes catalisadores e intensificação do estudo sobre a viabilidade econômica da instalação de uma planta industrial.

Sugere-se a inclusão contínua de artigos, em determinados intervalos de tempo, e utilização de outras plataformas como banco dados inicial para que se realce o acervo disposto e permita uma melhoria contínua do banco de dados formado.

\section{Agradecimento}

Agradeço à FAPES/CAPES pelo auxílio financeiro fornecido, possibilitando o desenvolvimento desta pesquisa.

\section{Referências}

ASSOCIAÇÃO BRASILEIRA DE EMPRESAS DE LIMPEZA PÚBLICA E RESÍDUOS ESPECIAIS (ABRELPE). Panorama dos resíduos sólidos no Brasil 2015, 2015.

BOtelho, L. L. R.; MACEDO, M.; GAUTHIER, F. O.; DUARTE, M. A. T. Revisão bibliométrica sobre mudança organizacional e aprendizagem gerencial em uma organização intensiva em conhecimento. XXV Encontro da associação nacional de pós-graduação e pesquisa em administração, 2011.

BREBU, M.; UCAR, S.; VASILE, C.; YANIK, J. Co-pyrolysis of pine cone with sinthetic polymers. Fuel, v. 89, p. 1911-1918, 2010.

BRIDGWATER, A. V. Review of fast pyrolysis of biomass and product upgrading. Biomass and bioenergy, v. 38, p. 68-94, 2012. 
ÇEPELIOĞULLAR, O.; PÜTUN, A. E. Thermal and kinetics behaviors of biomass and plastic wastes in co-pyrolisis. Energy conversion and management, v. 75, p. 263-270, 2013.

COLLOT, A. G.; ZHUO, Y.; DUGWELL, D. R., KANDIYOTI, R. Co-pyrolysis and cogasification of coal and biomass in bench-scale fixed-bed and fluidised bed reactors. Fuel, v. 78, p. 667-679, 1999.

CORNELISSEN, T.; YPERMAN, J.; REGGERS, G.; SCHREURS, S.; CARLEER, R. Flash co-pyrolysis of biomass and polylactic acid. Part I: influence of bio-oil yield and heating value. Fuel, v.87, p. 1031-1041, 2008.

CZERNIK, S.; BRIDGWATER, A. V. Overview of application of biomass fast pyrolysis oil. Energy e fuels, v. 18, p. 590-598, 2004.

DORADO, C.; MULLEN, C. A.; BOATENG, A. A. H-ZSM5 catalyzed co-pyrolysis of biomass and plastics. ACS sustainable chemistry e engineering, v. 2, p. 301-311, 2014.

GARCÌA-PÈREZ, M.; CHAALA, A.; YANG; J.; ROY, C. Co-pyrolysis of sugarcane bagasse with petroleum residue. Part I: thermogravimetric analysis. Fuel, v. 80, p. 1245-1258, 2001.

HOLLADAY, J. D.; HU, J.; KING, D. L.; WANG, Y. An overview of hydrogen production Technologies. Catalysis today, v. 139, p. 244-260, 2009.

HOROWITZ, H. H.; METZGER, G. A new analysis of thermogravimetric traces. Analytical chemistry, v. 35, n. 10, 1963.

INSTITUTO DE PESQUISA ECONÔMICA APLICADA (IPEA). Diagnóstico dos resíduos sólidos urbanos - relatório de pesquisa, Brasília, 2012.

KRERKKAIWAN, S.; FUSHIMI, C.; TSUTSUMI, A.; KUCHONTHARA, P. Synergetic effect during co-pyrolysis/gasification of biomass and sub-bituminous coal. Fuel processing technology, v. 115, p. 11-18, 2013. 
LEFÈVRE, M.; PROIETTI, E.; JAOUNEN, F.; DODELET, J.P. Iron-based catalysts with improved oxygen reduction activity in polymer electrolyte fuel cells. Science, v. 324, p. 71774, 2009.

LU, K. M.; LEE, W. J.; CHEN, W. H.; LIN, T. C. Thermogravimetric analysis and kinetics of co-pyrolysis of raw/torrefied wood and coal blends. Applied energy, v. 105, p. 57-65, 2013.

MA, F.; HANNA, M. A. Biodiesel production: a review. Bioresource technology, v. 70, p. 1$15,1999$.

MACEDO, M.; BOTELHO, L. L. R.; DUARTE, M. A. T. Revisão bibliométrica sobre a produção científica em aprendizagem gerencial. Revista gestão e sociedade, v. 4, n. 8, 2010.

MA, F.; HANNA, M. A. Biodiesel production: a review. Bioresource technology, v. 70, p. 1$15,1999$.

MARIN, N.; COLLURA, S.; SHARYPOV, V.I.; BEREGOVTSOVA, N.G.; BARYSHNIKOV, S.V.; KUTNETZOV, B. N.; CEBOLLA, V.; WEBER, J.V. Copyrolysis of wood biomass and synthetic polymer mixtures. Part II: characterisation of liquid phases. Journal of analytical and applied pyrolysis, v. 65, p. 41-55, 2002.

MOHAN, D.; PITTMAN Jr., C. U.; STEELE, P. H. Pyrolysis of wood/biomass for bio-oil: a critical review. Energy \& fuel, v. 20, p. 848-889, 2006.

ÖNAL, E.; UZUN, B. B.; PÜTÜN, A. E. Bio-oil production via co-pyrolysis of almond shell as biomass and high density polyethylene. Energy conversion and management, v. 78, p. 704-710, 2014.

PARADELA, F.; PINTO, F.; GULYURTLU, I.; CABRITA, I.; LAPA, N. Study of copyrolysis of biomass and plastic wastes. Clean technology environmental policy, v. 11, p. 115-122, 2009. 
PARK, D. K.; KIM, S. D.; LEE, S. H.; LEE, J. G. Co-pyrolysis characteristics of sawdust and coal blend in TGA and a fixed bed reactor. Bioresource technology, v. 101, p. 6151-6156, 2010.

SHARYPOV, V.I.; BEREGOVTSOVA, N. G.; KUZNETSOV, B. N.; MEMBRADO, L.; CEBOLLA, V. L.; MARIN, N.; WEBER, J.V. Copyrolysis of wood biomass and synthetic polymer mixtures. Part III: characterisation of heavy products. Journal of analytical and applied pyrolysis, v. 67, p. 325-340, 2003.

SHARYPOV, V.I.; MARIN, N.; BEREGOVTSOVA, N. G.; BARYSHNIKOV， S. V.; KUZNETSOV, B. N.; CEBOLLA, V. L.; WEBER, J.V. Copyrolysis of wood biomass and synthetic polymer mixtures. Part I: influence of experimental conditions on the evolution of solids, liquids and gases. Journal of analytical and applied pyrolysis, v. 64, p. 15-28, 2002.

TSANG, W.; HAMPSON, R. F. Chemical kinetic data base for combustion chemistry. Part I: methane and related compounds. Journal of physical and chemical reference data, v. 15, $\mathrm{n}$. 3, p. 1087-1279, 1986.

WONG, S.L.; NGADI, N.; ABDULLAH, T. A. T.; INUWA, I.M. Current state and future prospects of plastic waste as source of fuel: a review. Renewable and sustainable energy reviews, v. 50, p. 1167-1180, 2015.

XIA, T.; KOVOCHICH, M.; LIONG, M.; MÄDLER, L.; GILBERT, B.; SHI, H.; YEH, J. I.; ZINK, J. I.; NEL, A. E. Comparison of the mechanism of the toxicity of zinc oxide and cerium oxide nanoparticles based on dissolution and oxidative stress properties. ACS nano, v. 2, n. 10, p. 2121-2134, 2008.

YANG, H.; YAN, R.; CHEN, H.; LEE, D. H.; ZHENG, C. Characteristics of hemicellulose, cellulose and lignin pyrolysis. Fuel, v. 86, p. 1781-1788, 2007.

ZHANG, L.; XU, S.; ZHAO, W.; LIU, S. Co-pyrolysis of biomass and coal in free fall reactor. Fuel, v. 86, p. 353-359, 2007. 


\section{Res., Soc. Dev. 2019; 8(2):e1282585}

ISSN 2525-3409 | DOI: http://dx.doi.org/10.33448/rsd-v8i2.585

ZHOU, L.; WANG, Y.; HUANG, Q.; CAI, J. Thermogravimetric characteristics and kinetic of plastic and biomass blend co-pyrolysis. Fuel processing technology, v. 87, p. 963969, 2006.

ZHU, W.; SONG, W.; LIN, W. Catalytic gasification of char from co-pyrolysis of coal and biomass. Fuel processing technology, v. 89, p. 890-896, 896, 2008. 